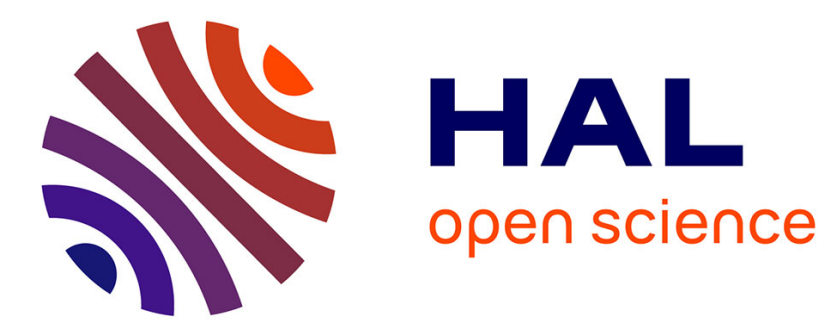

\title{
Voir, juger, découvrir : la place du regard dans la Querelle du Cid
}

Clotilde Thouret

\section{To cite this version:}

Clotilde Thouret. Voir, juger, découvrir: la place du regard dans la Querelle du Cid. Littératures classiques, 2013, L'œil classique. Regards croisés sur le XVIIe siècle, 82, pp.99-111. hal-02903447

\section{HAL Id: hal-02903447 \\ https://hal.univ-lorraine.fr/hal-02903447}

Submitted on 21 Jul 2020

HAL is a multi-disciplinary open access archive for the deposit and dissemination of scientific research documents, whether they are published or not. The documents may come from teaching and research institutions in France or abroad, or from public or private research centers.
L'archive ouverte pluridisciplinaire HAL, est destinée au dépôt et à la diffusion de documents scientifiques de niveau recherche, publiés ou non, émanant des établissements d'enseignement et de recherche français ou étrangers, des laboratoires publics ou privés. 


\section{Clotilde Thouret \\ Voir, juger, découvrir : \\ la place du regard dans la Querelle du Cid}

La Querelle du Cid s'ouvre et se clôt sur une mise en cause du regard des spectateurs : dans les Observations sur le Cid, Scudéry déplore les « beautés d'illusion » qui ont abusé le peuple comme les « honnêtes gens », et dans sa réponse à Balzac, il évoque des « beautés [de la poésie] que tous les yeux n'aperçoivent pas 1 ». L'œil des spectateurs peut donc être trompé ou aveuglé - et tous les yeux n'ont pas la même capacité de discernement. Scudéry est loin d'être le seul dans la controverse à construire son argumentation, ou une partie de celle-ci, sur ce qui a été vu lors de la représentation, sur la perception du spectateur ou sur ce que la pièce a exposé à la vue du public. Depuis le début de la Querelle, et probablement depuis les premières représentations, jusqu'à sa fin, le regard ne cesse d'occuper, et aussi d'ordonner, à des degrés divers, les prises de position.

Qu'il fonctionne comme un nœud polémique transparaît notamment dans le titre d'une publication anonyme : Le Souhait du Cid en faveur de Scudéry : une paire de lunettes pour faire mieux ses Observations. Certes le terme de «lunette » s'est déjà rencontré dans la littérature de controverse2, mais il fait affleurer dans le titre du poète dramaturge le sème du regard ou de la vision, qui, dans l'usage d' " observation », demeure en retrait par rapport à l'obéissance à une règle ou à la remarque que l'on fait sur une chose ou sur un écrit. Au-delà de cette pointe à laquelle il ne faut peut-être pas donner trop d'importance, toute une partie des débats tourne autour de la vision, de ce qu'on voit et perçoit, mais aussi de ce que l'on peut montrer (au théâtre, ou à la cour d'Espagne) ou de ce qui doit être mis en scène.

Cette forte présence du regard dans les textes pourrait n'être qu'un effet de la logique entropique de la Querelle, les défenseurs reprenant un à un les arguments des attaquants et réciproquement. Loin de signaler un point de discorde réel, la centralité de la question de la perception serait seulement un effet de la dynamique rhétorique propre à la polémique. Cependant, comme on le verra, le regard est toujours, explicitement ou non, articulé à la question du jugement, et par là il touche à une question majeure qui sous-tend et explique la Querelle3. Aussi peut-on supposer que la Querelle du Cid fonctionne comme un moment de mise au point théorique sur le regard du spectateur, comme un événement révélateur d'un état des pensées et des représentations de la vision.

\section{L'œil abusé par un éclat trompeur}

Les premières lignes des Observations assignent au regard un rôle décisif dans le succès, jugé usurpé et injustifié, de la pièce :

Il est de certaines pièces, comme de certains animaux qui sont en la nature, qui de loin semblent des étoiles, et qui de près ne sont que vermisseaux. Tout ce qui brille n'est pas toujours précieux ; on voit des beautés d'illusion, comme des beautés effectives, et souvent l'apparence du bien se fait prendre pour le bien même. Aussi ne m'étonné-je pas beaucoup que le peuple, qui porte le jugement dans les yeux, se laisse tromper par celui de tous les sens le plus facile à décevoir. Mais que cette vapeur grossière qui se forme dans le parterre, ait pu s'élever jusqu'aux galeries, et qu'un fantôme ait abusé le savoir comme l'ignorance, et la cour aussi bien que le bourgeois, j'avoue que ce prodige m'étonne, et que ce n'est qu'en ce bizarre événement que je trouve Le Cid merveilleux.4

Le point de départ de l'argumentation de Scudéry est l'erreur provoquée par la vision de loin ou par une apparence trompeuse : on ne peut pas se fier au seul regard pour discerner la vérité. L'idée, éminemment baroque et dans laquelle on pourrait voir un retournement ironique de L'Illusion comique, est ensuite appliquée à la représentation de la tragi-comédie de Corneille et articulée à la hiérarchie sociale par sa transposition dans l'architecture de la salle de théâtre. Le défaut de la vision, la propension du regard à être trompé, concerne avant

1 Cette lettre est publiée en 1638, chez Augustin Courbé à Paris, avec celle que Balzac avait adressée à Scudéry et la lettre de ce dernier « à ces Messieurs de l'Académie française, sur le Jugement qu'ils ont fait du Cid». La citation se trouve p. 1106 de l'édition procurée par Jean-Marc Civardi (La Querelle du Cid, Paris, Champion, 2004); sauf mention contraire, pour les textes de la Querelle, je me réfère à cet ouvrage et je les indiquerai de la manière suivante: Querelle, p. 1106. J'en ai modernisé l'orthographe, la typographie et la ponctuation.

2 J.-M. Civardi le signale en note (Querelle, p. 663).

3 Comme l'a montré Hélène Merlin-Kajman dans Public et littérature en France au XVII siècle ([1994], Paris, Les Belles Lettres, 2004), la Querelle du Cid cristallise des pensées opposées sur le jugement légitime, et avec elles, des conceptions distinctes du public.

4 Querelle, p. 367-368. Sur ces lignes, voir l'analyse d'H. Merlin-Kajman, op. cit., p. 171-176, que je reprends ici. 
tout le peuple qui "porte le jugement dans les yeux » et est incapable de mettre à distance le phénomène spectaculaire afin de faire réflexion sur lui. Plus encore, l'enthousiasme du parterre a été tel qu'il a troublé la perception des honnêtes gens : ceux-ci se sont trouvés entraînés dans l'illusion malgré leur esprit et se sont laissé prendre aux beautés fallacieuses de la représentation, par un phénomène quasi physique 5. Les applaudissements qui ont salué la pièce de Corneille ne sont donc pas un jugement, du moins pas un jugement légitime.

La suite du texte reprendra cette première idée sous d'autres formes, en mobilisant le champ sémantique de l'illusion et des apparences trompeuses6. Surtout, les mots avec lesquels Scudéry formule son attaque posent les termes du débat car ils seront repris, parfois à l'identique, tout au long de la Querelle. Ainsi, l'explication du succès par un "éclat trompeur » revient plusieurs fois, par exemple dans l'Épître familière du sieur Mairet au sieur Corneille sur la tragi-comédie du Cid où Mairet parle des " beautés apparentes et fantastiques » de la pièce, de son « faux éclat », et la compare à de la quincaillerie prise pour de l'or7.

Un argument de poids vient parfois soutenir l'accusation : c'est le spectacle et en particulier les acteurs qui sont à l'origine des applaudissements et qui ont fait la beauté de la pièce. L'auteur du Discours à Cliton le sousentend lorsqu'il déplore la pratique des comédiens qui mettent en avant leur art et leur personne plutôt que le héros qu'ils incarnent8; Mairet le formule plus explicitement dans son Épître familière en suggérant à Corneille d'orner la publication de gravures en taille douce pour ajouter au texte « les gestes, le ton de voix, la bonne mine et les beaux habits de ceux et celles qui les [ses nobles journées] ont si bien représentées, puisqu['il pouvait] juger qu'ils faisaient la meilleure partie de la beauté de [son] ouvrage9 ». Scudéry avait lui-même fait fond sur le clinquant illusoire de la représentation dans sa lettre à l'Académie pour demander l'intervention de celle-ci et justifier un jugement s'appuyant avant tout sur le texte imprimé :

[La pièce du $\mathrm{Cid}$ ] n'avait de beautés que celle que ces agréables trompeurs qui la représentaient lui avaient prêtée, et [...] Mondory, la Villiers et leurs compagnons n'étant pas dans le livre comme sur le théâtre, le Cid imprimé n'était plus le Cid que l'on a cru voir.10

Commentant ces lignes, Hélène Merlin-Kajman souligne la différence, fondamentale dans la position de Scudéry, entre ce que l'on voit lors d'une représentation et ce qui est perçu par la lecture savante du texte : « là où l'espace public du théâtre publie en aveuglant, l'impression, elle, publie en éclaircissant les défauts du Cid11 ». On voit combien la question de la vision est fortement liée à celle du jugement et de sa légitimité, et qu'aux yeux des critiques de la pièce seuls les yeux de l'esprit peuvent donner naissance à un jugement véritable, inaccessible par conséquent au public du théâtre.

L'aveuglement des spectateurs ignorants nourrit enfin une comparaison religieuse aux résonances d'autant plus fortes que le souvenir des guerres de religion était proche : les partisans de la pièce et ceux qui l'ont applaudie sont comme des hérétiques égarés qu'il faut ramener à la raison et à la vérité. L'auteur de la Lettre $\grave{a}{ }^{* * *}$ sous le nom d'Ariste dit avoir pris « la résolution de guérir ces idolâtres de leur aveuglement» et Mairet reprendra la métaphore dans son Épître, en évoquant le peuple et les femmes, ces "idolâtres », éblouis par le faux éclat de la pièce avant d'être convertis par les remarques du « clairvoyant observateur12 ». Tous les regards n'ont pas la même capacité ni la même compétence : à la vue aisément abusée ou aveuglée du peuple s'oppose le regard aiguisé des doctes, éclairé par leur esprit et leur savoir. L'opposition structure pratiquement tous les textes des détracteurs du Cid : elle vient d'autant plus facilement sous leur plume que le poème dramatique donne lieu à un spectacle, donc à une réception sensible immédiatement partagée.

5 Furetière donne comme définition de «vapeur»: "Parties subtiles d'un corps humide, qu'une chaleur médiocre élève et ne peut dissiper», et le dictionnaire de l'Académie (1694) : "Fumée qui s'élève des choses humides »; " vapeur » désigne à cette époque une " émanation visible », une " exhalaison de corps liquides ou solides » (Dictionnaire historique de la langue française).

6 Par exemple au sujet de l'acte III, si central dans le succès de la pièce : «Insensiblement nous voici arrivés au troisième acte, qui est celui qui a fait battre des mains à tant de monde, crier miracle à tous ceux qui ne savent pas discerner le bon or d'avec l'alchimie, et qui seul a fait la fausse réputation du Cid » (Querelle, p. 396). Voir aussi les «belles couleurs qui s'effacent en l'air presqu'aussitôt que le soleil en a fait la riche et trompeuse impression sur la nue » (p. 370).

7 Querelle, p. 801, 808 et 806. J.-M. Civardi dans sa présentation relève d'autres éléments qui nourrissent la critique du Cid comme « enchantement pernicieux » (Querelle, p. 88-91).

8 Querelle, p. 620-621.

9 Querelle, p. 804. «Nobles journées » désigne la pièce, et a le sens de «grandes batailles », ou "grands exploits »; Mairet se sert ironiquement de l'expression pour désigner le texte à succès de Corneille, que, trop impatient, il n'a pas repris avant de le faire imprimer; c'est en outre une citation implicite d'un vers de l'Infante (v. 543) que Scudéry avait justement repris dans ses Observations (Querelle, p. 415).

10 Querelle, p. 566 ; Mondory interprétait le rôle de Rodrigue, et la Villiers, celui de Chimène.

11 H. Merlin-Kajman, op. cit., p. 175.

12 Querelle, p. 732 et 808. 


\section{Le regard juge}

Avant d'envisager ce que cette pensée de la vision doit aux conceptions théâtrales que Scudéry et Chapelain élaborent à l'occasion de la Querelle, il convient de s'arrêter sur les réponses des défenseurs, qui permettent d'identifier d'une part, la portée polémique de l'argument de l'illusion et d'autre part, une autre dynamique du regard. La Défense du Cid dénonce ainsi le caractère fallacieux de la stigmatisation de la vue comme le plus trompeur de tous les sens ; tous les sens sont en effet susceptibles d'être abusés :

Et faisant le philosophe, [l'observateur] dit que le peuple se laisse tromper par celui de tous les sens le plus facile à décevoir; il ferait plus que tous les maîtres du métier, s'il nous pouvait montrer qu'un sens soit plus avantagé que l'autre contre la tromperie, tous sont en état également de l'être, et de ne l'être pas, si l'on prend la raison commune de leur objet, comme pour la vue, la couleur en général, pour l'ouïe, la voix et les sons.13

De fait, Scudéry se prononce contre l'opinion commune et la tradition, qui font de l'œil le plus noble de tous les sens et la source d'un émerveillement toujours renouvelé pour ses beautés et ses merveilles14. Le "paradoxe» scudérien peut être interprété de deux manières, qui ne sont pas nécessairement exclusives l'une de l'autre : ou bien comme un argument d'autorité caractéristique d'une mauvaise foi d'auteur envieux, ou bien comme l'effet d'une méfiance à l'égard du spectacle et de ses compromissions avec le sensible, méfiance que partage avec les détracteurs du théâtre une partie des théoriciens et des dramaturges 15 .

La faiblesse argumentative de la position de Scudéry est aussi soulignée par l'auteur du Souhait du Cid, qui retourne contre l'observateur, avec une certaine vigueur non dénuée d'esprit, la dévalorisation du jugement des spectateurs. D'une part, c'est Scudéry qui est dans les ténèbres, aveuglé qu'il est par son orgueil de docte16, d'autre part, il est ridicule d'invoquer un jugement paralysé par les sens lors de la représentation, car cela suppose un dédoublement peu crédible, entre les sens et l'esprit, entre Scudéry au théâtre et Scudéry dans son cabinet17. Le succès du Cid est fondé, il ne tient pas à l'infériorité du jugement des spectateurs, ou plus exactement, à sa résorption dans le seul organe de la vue qui serait sans profondeur ni capacité de discernement.

C'est aussi ce qu'exprime l'auteur du Jugement du Cid, composé par un bourgeois de Paris, Marguillier de sa paroisse, en faisant intervenir les affects suscités par la représentation d'une manière tout à fait remarquable :

Je me suis enfin résolu, attendant le jugement de l'Académie, de faire voir le mien, qui est, ce me semble, le sentiment des honnêtes gens d'entre le peuple ; [...] et [j'ai voulu montrer] que le sens commun n'est pas entièrement banni de la tête de ceux qui ne sont ni savants, ni auteurs.

Je n'ai jamais lu Aristote, et ne sais point les règles du théâtre, mais je règle le mérite des pièces selon le plaisir que j'y reçois. Celle-ci a je ne sais quoi de charmant dans son accident extraordinaire ; et il n'y a personne qui après avoir vu le mariage résolu des deux amants, n'entre en de grandes craintes pour eux aussitôt que les pères commencent à se quereller; qui ne soit ému voyant l'affront que reçoit Don Diègue; qui ne soit troublé voyant le commandement qu'il fait à son fils de le venger; et qui ne s'attendrisse de pitié voyant le combat en Rodrigue entre son honneur et son amour. Mais jamais rien n'a plus transporté les spectateurs qu'alors que Rodrigue ayant tué le Comte, vient chez Chimène lui demander la mort, et met le même combat en son esprit entre son amour et son honneur. Ces deux combats, également grands dans les deux principaux personnages, et qui entretiennent toute la pièce, donnent tant de pitié et de plaisir ensemble, que jusques ici rien ne s'était vu qui eût tant attaché l'attention. 18

La revendication d'un sens commun, et d'un jugement solide et légitime qui serait celui du public, passe par la

13 Querelle, p. 464-465.

14 Voir C. Havelange, De l'œil et du monde. Une histoire du regard au seuil de la modernité, Paris, Fayard, 1998, chap. 2 .

15 Sur ce point, sur lequel j'aurai l'occasion de revenir, voir M. Vuillermoz, « Le spectacle en procès. Réflexions sur le sens des réquisitoires chez les théoriciens du théâtre à l'âge classique », R.H.L.F., 2006, $\mathrm{n}^{\circ} 3$, p. 629-641.

16 «Les deux soleils d'Aristote ne sauraient donner assez de clarté à un aveugle qui se trouve dans les ténèbres dont l'esprit et jugement de Scudéry est environné ; pensant avoir trop de lumière il n'a vu goutte [...]» (Querelle, p. 672).

17 La présomption de l'auteur du Cid aurait donné à Scudéry « la curiosité de regarder de près ce qu'il n'avait vu que de loin, et d'appeler de ses oreilles et de ses yeux à son esprit, à qui le ton de la voix et le geste donnant du divertissement ne permirent pas durant le cours de l'action de juger exactement de ce qui s'y passait » (Querelle, p. 665). La formule renvoie à celle de Scudéry qui, dans les Observations, disait suivre l'exemple d'un Macédonien qui en « appela de Philippe préoccupé à Philippe mieux informé » (Querelle, p. 368-369).

18 Querelle, p. 779-781 (je souligne). 
mise en avant des passions éprouvées par les spectateurs lors de la représentation et qui ont donné naissance à leur plaisir. Tous les événements de la pièce sont diffractés par le prisme des émotions : celles-ci fonctionnent comme la pierre de touche de la valeur de l'œuvre, comme des signes fidèles et vrais du bien-fondé de la faveur que $L e C i d$ a reçue du public. Or c'est bien la perception visuelle qui fait naître la crainte, le trouble, la pitié, l'attention. La répétition du verbe « voir » renvoie très directement à la locution de Scudéry : le peuple porte bien « le jugement dans les yeux », mais son regard n'est pas trompé ; l'œil du spectateur n'est pas une surface sur laquelle défilent des ombres trompeuses, il est l'organe qui met en rapport le spectateur avec l'action sur la scène, il est le sens qui intervient dans la formation d'affects qui fondent tout à la fois le plaisir et le jugement (sur les personnages comme sur la pièce), il est le truchement sensible par lequel passe l'interaction de l'âme avec le monde (ici, le spectacle). En revanche, contrairement à ce que laisse entendre la formule des Observations, il y a une médiation entre les yeux et le jugement : elle consiste dans les émotions, par lesquelles s'établit la relation du spectateur avec les personnages. Ces lignes du Marguillier laissent deviner un regard actif, en opposition radicale avec le regard passif présupposé par Scudéry et par Chapelain.

\section{La passivité du regard : un point d'appui de l'utilité morale et politique du théâtre}

Mise en avant par les deux auteurs, cette passivité de l'organe contraste avec l'activité du jugement métaphorisée par les yeux de l'âme. On l'a vu, une partie du propos de Scudéry consiste à établir une distinction claire entre la réaction du peuple dans la salle de théâtre, entièrement soumise aux sens, et la réflexion de l'observateur ou des messieurs de l'Académie, dans le cabinet face au texte imprimé19. Même lors de la représentation, les honnêtes gens et les doctes ne s'arrêtent pas à la seule perception sensible et font intervenir leur esprit : ainsi la présence de Rodrigue chez Chimène à l'acte III « donne de l'horreur à tous les judicieux qui le voient et qui savent que [le] corps [du Comte] est encore dans la maison20».

Surtout, la passivité de l'œil fonde théoriquement la pensée de l'utilité morale et politique du théâtre que Scudéry puis Chapelain sont amenés à formuler à l'occasion de la Querelle, notamment afin d'étayer leurs critiques de la pièce et en particulier celle du personnage de Chimène ; sans être exactement identiques, leurs positions font appel à la vision du spectacle de manière similaire. Le passage des Observations qui suit la condamnation du mariage ordonné par le roi et précède celle, si violente, des mœurs de l'héroïne, est particulièrement révélateur :

Et pour connaître cette vérité [que la pièce choque les bonnes mœurs], il faut savoir que le poème de théâtre fut inventé pour instruire en divertissant; et que c'est sous cet agréable habit que se déguise la Philosophie, de peur de paraître trop austère aux yeux $d u$ monde, et par lui (s'il faut ainsi dire) qu'elle semble dorer les pilules, afin qu'on les prenne sans répugnance, et qu'on se trouve guéri, presque sans avoir connu le remède. Aussi ne manque-t-elle jamais de nous montrer sur la scène la vertu récompensée et le vice toujours puni. Que si quelquefois l'on y voit les méchants prospérer et les gens de bien persécutés, la face des choses, ne manquant point de changer, à la fin de la représentation, ne manque pas aussi de faire voir le triomphe des innocents et le supplice des coupables ; et c'est ainsi qu'insensiblement, on nous imprime en l'âme l'horreur du vice et l'amour de la vertu. 21

De même que l'on revêt un beau costume, les beautés du poème dramatique et le plaisir qu'elles procurent recouvrent l'instruction morale et politique; celle-ci pénètre alors plus facilement dans l'âme du spectateur et « s'y imprime » sans qu'il s'en aperçoive. La vue se fait ainsi adjuvant « inconscient» de la conduite des bons sentiments dans l'âme du spectateur; sa faiblesse - sa passivité - peut donc devenir un atout. L'œil se trouve de nouveau abusé ici mais l'effet est inverse de celui du Cid, puisque le spectateur venu au théâtre pour se divertir reçoit sans en avoir conscience ( insensiblement») des leçons morales22. La vue est toujours ce sens trompeur qui s'arrête à la surface des choses (la dorure), mais on peut le tromper à bon escient, par une belle représentation des vices punis et de la vertu récompensée.

L'idée était déjà présente dans la Lettre sur la règle des vingt-quatre heures, lorsque Chapelain cherche à fonder en raison les règles et en particulier celle de l'unité d'action : l'œil étant un organe fini, ses capacités limitées de perception obligent le peintre comme le poète dramatique à réduire la quantité d'action représentée pour conserver la créance du spectateur. Par cet aménagement, on conduit « l'œil surpris à se tromper lui-même

19 Voir le texte cité ci-dessus (Querelle, p. 566).

20 Querelle, p. 396 (je souligne).

21 Querelle, p. 383-384 (je souligne). Furetière : «Dorer la pilule c'est faire paraître une chose plus belle qu'elle n'est, faire avaler quelque amertume, quelque chose de fâcheux, en l'adoucissant par de belles paroles. » 22 Sur la tromperie comme ressort de l'effet moral du théâtre et sur l'influence de la théorie italienne (en particulier de la querelle du Pastor fido) sur l'instrumentalisation politique du plaisir du spectateur, voir D. Blocker, «Jean Chapelain et les Lumières de Padoue. L'héritage italien dans les débats français sur l'utilité du théâtre (1586-1640) », Littératures classiques, n 37 (De l'utilité de la littérature, dir. A. Viala), 1999, p. 97-116. 
pour son profit», car le spectateur pourra ainsi être touché «par l'opinion de la vérité23 ». L'ambivalence du regard, source d'erreur et de propagation des vices mais aussi point d'appui de l'utilité morale et politique du théâtre, se trouvera de nouveau exprimée dans la version définitive des Sentiments de l'Académie :

Et certes il n'est pas croyable qu'un plaisir puisse être contraire au bon sens, si ce n'est le plaisir de quelque goût dépravé comme est celui qui fait aimer les aigreurs et les amertumes. Il n'est pas ici question de satisfaire les libertins et les vicieux qui ne font que rire des adultères et des incestes, et qui ne se soucient pas de voir violer les lois de la nature pourvu qu'ils se divertissent. Il n'est pas question de plaire à ceux qui regardent toutes choses d'un æil ignorant ou barbare, et qui ne seraient pas moins touchés de voir affliger une Clytemnestre qu'une Pénélope. Les mauvais exemples sont contagieux, même sur les théâtres; les feintes représentations ne causent que trop de véritables crimes, et il y a grand péril à divertir le peuple par des plaisirs qui peuvent produire un jour des douleurs publiques. Il nous faut bien garder d'accoutumer ni ses yeux ni ses oreilles à des actions qu'il doit ignorer et de lui apprendre tantôt la cruauté, et tantôt la perfidie, si nous ne lui en apprenons en même temps la punition, et si au retour de ces spectacles il ne remporte du moins un peu de crainte parmi beaucoup de contentement.24

L'utilité morale du théâtre est conditionnée par la punition des crimes car «l'œil ignorant ou barbare »-celui du peuple - posé sur un mauvais exemple peut être la source d'une accoutumance aux vices, ces derniers se transmettant à l'âme par l'intermédiaire de la vue25. Cependant, ce processus de transmission, qui passe par le regard et produit une réponse affective, peut être mobilisé au profit du gouvernement de l'État et de la morale de ses sujets. Il y a un bon et un mauvais usage esthético-éthique du sens visuel ; il importe donc de maitriser par les règles les effets produits par lui sur les spectateurs.

Ces analyses appellent deux remarques. D'abord, la représentation de la vision comme sens passif et susceptible d'erreur permet à Scudéry et Chapelain de faire une distinction majeure entre les spectateurs qui reçoivent passivement ce qu'on leur montre et ceux qui peuvent faire réflexion sur leur perception, entre ceux qui sont capables de plaisir (plaisir qu'il s'agit de bien orienter vers le profit moral) et ceux qui sont capables de jugement ; leur partage du sensible correspond bien à une hiérarchie des capacités26. Ensuite, leurs élaborations théoriques s'appuient sur un savoir optique nouveau, ou plutôt sur un certain usage de ce savoir, puisqu'il se trouve placé dans l'orbite du pouvoir et inscrit dans une hiérarchie sociale claire : leurs démonstrations s'adossent en effet aux récentes découvertes optiques et notamment à la transposition de la perspective à la représentation dramatique; dans cet «ordre nouveau du regard», l'œil fonctionne comme un témoin et le cerveau (quand il en a la capacité) interprète les images que l'œil lui transmet27.

\section{Le spectateur, Don Fernand : un regard qui découvre}

À l'inverse, si l'on suit l'analyse de Starobinski dans $L$ 'Eil vivant, le théâtre de Corneille serait plutôt du côté de «l'ordre ancien du regard», qui se caractérise par un œil « dans le monde28 » et par un regard capable d'une action à distance. Le critique le dit avant tout de l'univers fictionnel constitué par les pièces mais l'étend aussi à la création dramatique. Cette efficace du regard semble d'ailleurs animer la Querelle elle-même : le scandale et le succès de la tragi-comédie tient à ce qu'elle fait voir, à ce qu'elle place sous le regard du spectateur, et au premier chef l'amour de Chimène pour Rodrigue après la mort du comte.

Les analyses qu'H. Merlin-Kajman a consacrées à la pièce ont montré ce que le duo de l'acte III entre

23 J. Chapelain, «Lettre sur la règle des vingt-quatre heures », dans Opuscules critiques, éd. A. C. Hunter revue par A. Duprat, Genève, Droz, 2007, p. 225.

24 Querelle, p. 937-938 (je souligne). Ces lignes ne se trouvent pas dans la première version du texte et participent du durcissement du propos qui caractérise la version finalement retenue et publiée.

25 Plus tard, les moralistes comme Nicole et Malebranche expliqueront cette action sur le corps et sa mécanique (voir E. Koch, The Aesthetic Body. Passion, Sensibility, and Corporeality in Seventeenth-Century France, Newark, University of Delaware Press, 2008, p. 86-130) ; ainsi, partisans (doctes) et détracteurs du théâtre partagent les mêmes présupposés mais les seconds ne retiennent que les effets néfastes produits par la dimension visible du spectacle.

26 Jacques Rancière l'a mis en lumière pour ce qu'il appelle le « régime représentatif » des arts ; il convient ici de préciser que ce n'est pas la seule position dans la Querelle: dès le XVII ${ }^{\mathrm{e}}$ siècle, ce partage est donc concurrencé par d'autres.

27 Sur ces transformations, voir F. Siguret, L'Eil surpris. Perception et représentation dans la première moitié $d u$ XVII siècle [1985], Paris, Klincksieck, 1993, p. 7-9 et p. 16-24. C. Havelange, à qui j'emprunte la formule, insiste sur la présence d'une médiation qui s'installe entre l'œil et le monde : un voile, un tableau, une lunette, un scalpel, une image objectivante qui instrumente le regard (op. cit., chap. 8 et 9).

28 Dans ce régime ancien du voir, le regard fait exister et «signifie tout un système de réciprocités effectives entre le sujet voyant et ce qui est regardé » (C. Havelange, op. cit., p. 238). 
Rodrigue et Chimène pouvait avoir de fascinant pour les spectateurs de l'époque29 : tant l'épée de Rodrigue, qui fonctionne comme un véritable « coup visuel », que l'aveu d'amour de Chimène, dont elle fait un principe de sa gloire, constituent le caractère extraordinaire de ce moment. La scène découvrait alors au spectateur que « derrière le visage public peut toujours se dissimuler un autre visage trahi par ses affects »; aussi excitait-elle une «formidable curiosité30». Que cette contradiction entre les exigences du public et le sentiment privé s'expose sur une scène de théâtre n'est évidemment pas sans importance ni sans effet : à travers ce dialogue, Chimène et Rodrigue « accordent de l'espace, de la visibilité, du spectacle mutuel, à leur particulier31».

L'attitude du roi joue également un rôle dans le scandale : l'ordre du mariage donne à l'amour de Chimène un accomplissement public et c'est en parlant de ce dénouement que Chapelain juge Le Cid « de trop mauvais exemple pour l'exposer à la vue du peuple32 ». Scudéry quant à lui avait stigmatisé la ruse qui fonde la décision du roi comme indigne de son rang et de sa fonction. Afin d' «éprouver33 » le sentiment de Chimène, le roi lui tend un «piège » : après la victoire contre les Maures, elle vient une nouvelle fois demander la tête du meurtrier de son père ; s'accordant avec Don Diègue ( “Contrefaites le triste34 »), Don Fernand lui annonce la mort de Rodrigue ; la pâmoison de la jeune fille, même si elle se récrie par la suite, ne laisse plus de doute sur son amour.

L'événement qui a lieu alors sur la scène se formule en termes de vision et le regard intervient d'une manière décisive dans le déroulement de l'action. Comme l'analyse Scudéry, cette " méchante finesse » fait "découvrir aux yeux de toute la Cour, par un évanouissement l'infâme passion qui la possède35 ». L'auteur du Souhait du Cid, en objectant à cette observation, reprendra le terme («Fernand use d'une très grande prudence pour découvrir les mouvements de l'esprit de Chimène ») et après lui, Chapelain36. Le texte dramatique lui-même attire l'attention sur la dynamique du regard qui observe en même temps qu'il révèle :
LE ROI
Voyez comme déjà sa couleur est changée.
DON DIEGUE
Mais voyez qu'elle pâme, et d'un amour parfait
Dans cette pâmoison, Sire, admirez l'effet, [...]37

La scène s'inscrit de fait dans le mouvement de dévoilement de la passion de Chimène qui caractérise la pièce depuis la mort du Comte ; elle en est une des étapes : après que les spectateurs en ont été les témoins à l'acte III, elle est ici vérifiée par le roi et montrée à la Cour, avant que l'héroïne ne la laisse elle-même « éclater » et paraître aux yeux de tous de son propre mouvement38.

Finalement, le roi révèle publiquement cet amour en le rendant visible dans l'espace du palais, aux yeux de toute la Cour, et alors même qu'il occupe une position de juge ; cette position est en effet celle que lui donne la requête de Chimène, celle que lui assigne la scénographie qui reproduit la configuration de l'accusation (acte II, sc. 7) et celle que lui reconnaîtra Corneille dans l'examen de Clitandre. Le regard est ainsi intégré à l'exercice royal de la justice : l'œil de Don Fernand découvre le sentiment de Chimène, au sens où ce qu'il voit confirme ce qu'il soupçonnait; en outre, il le découvre aux autres sujets et par là le fait exister. Car exposer aux regards revient ici non seulement à dévoiler, mais aussi à autoriser, et donc à reconnaître : en le destinant au mariage, le roi donne à cet amour une place dans l'espace public, il le reconnaît comme acceptable voire comme utile au bien commun puisqu'il lui gardera le nouveau rempart de la Castille, Rodrigue39.

Comme l'avait fait (sans le vouloir) Rodrigue lorsqu'il écoutait pleurer Chimène derrière la tenture (acte III, sc. 3), le roi s'est donné la position privilégiée des spectateurs, celle qui permet de voir dans les appartements privés. La très brève illusion (ou feinte) mise en place avec l'aide de Don Diègue souligne d'ailleurs le parallèle avec le théâtre. Son regard, rejoignant alors celui du public, a changé le statut de cette passion scandaleuse,

29 Voir en particulier L'Absolutisme dans les lettres et la théorie des deux corps. Passions et politique, Paris, Champion, 2000, chap. IV et VII.

30 Ibid., p. 124.

31 H. Merlin-Kajman, «La "scène publique" dans L'Illusion comique et dans Le Cid: fanfaronnades et bravades », Littératures, $\mathrm{n}^{\circ}$ 45, automne 2001, p. 61.

32 J. Chapelain, Sentiments de l'Académie, dans Opuscules critiques, éd. cit., p. 289.

33 Le mot est celui du roi (acte IV, sc. 4, v. 1346) et est repris par Scudéry (Querelle, p. 401).

34 Acte IV, sc. 4, v. 1347.

35 Querelle, p. 401-402 (je souligne).

36 Querelle, p. 682, et p. 990 : «Car cela se pourrait bien défendre, par [l'exemple] de plusieurs grands princes qui n'ont pas fait difficulté d'user de feintise dans leurs jugements, quand ils ont voulu découvrir une vérité cachée. »

37 Acte IV, sc. 5, v. 1352-1354.

38 Acte V, sc. 5, v. 1719 : «Éclate, mon amour, tu n'as plus rien à craindre, [...]»; acte V, sc. 7, v. 1828 : «Mon amour a paru, je ne m'en puis dédire, $[. .$.$] »$

39 L'Infante a signalé aux spectateurs cet aspect de l'alliance lorsque, dans une scène précédente, elle a reproché à Chimène de «poursuivre [...] la ruine publique » en cherchant à se venger (acte IV, sc. 2, v. 1192). 
trouvant ainsi une issue extraordinaire à cette situation exceptionnelle. Qu'on se place du point de vue du pouvoir politique ou du côté de la pratique dramatique, la pièce de Corneille instaure donc, voire institue, un regard qui découvre, dans les deux sens du terme; ce regard est celui des spectateurs tout autant que celui des personnages et en particulier celui du roi - d'où la centralité de la vision dans les débats.

La Querelle du Cid voit donc s'opposer deux représentations très différentes du regard du spectateur, et leur cohérence apparaît mieux encore si l'on reconsidère la tragi-comédie elle-même à la lumière de ce nœud polémique. Sans doute radicalisés par les débats, ces deux imaginaires de la vision coïncident avec les deux camps de la controverse - et de façon un peu surprenante, la modernité scientifique ne se trouve pas du côté de la modernité poétique. À l'œil susceptible d'être trompé par les apparences invoqué par les adversaires de Corneille s'oppose le regard source de plaisir et de vérité mis en avant par ses partisans. Les premiers conçoivent la vue comme une perception passive qui, si elle peut être mise à profit dans une manipulation poétique à des fins morales et politiques, ne peut en aucun cas fonder le véritable jugement; les seconds quant à eux l'inscrivent dans une dynamique affective qui légitime au contraire le jugement et constitue une forme nouvelle de public. À cet égard, la logique interne de la pièce rejoint la logique de la représentation théâtrale, et le regard de Don Fernand celui des spectateurs : véritable acte social, il découvre l'affect passionnel privé et, en l'articulant avec d'autres émotions (l'admiration en particulier), l'autorise et la reconnaît. Art des circonstances, le théâtre de Corneille, comme la justice de Don Fernand, est aussi un art de la composition.

Clotilde Thouret Université Paris-Sorbonne 